\section{CABG (n=83)}

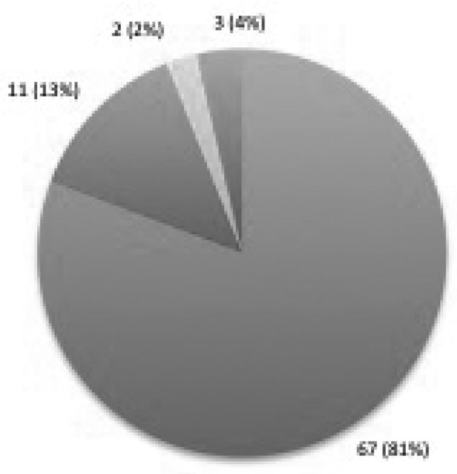

PCI (n-19)

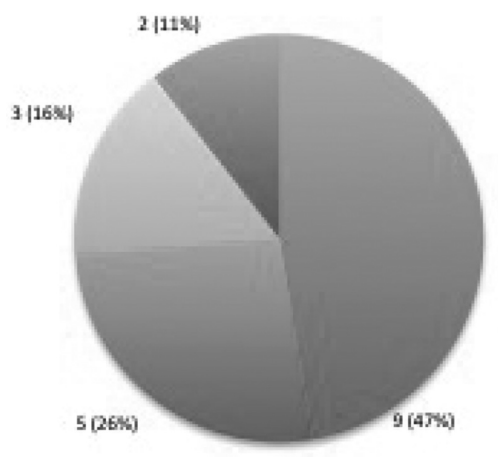

Abstract 33 Figure 2 Reasons for delay $>14$ days for CABG and $\mathrm{PCI}$

pathway to both surgical and percutaneous revascularisation for this patient group are needed.

\section{DO CENTRES THAT USUALLY PERFORM PERCUTANEOUS CORONARY INTERVENTION TRANS-RADIALLY HAVE INFERIOR OUTCOMES WHEN OPERATING TRANS- FEMORALLY?}

${ }^{1}$ William Hulme* ${ }^{1}$ Matthew Sperrin, ${ }^{1}$ Evan Kontopantelis, ${ }^{2}$ Peter Ludman, ${ }^{3}$ Mark de Belder, ${ }^{4}$ James Nolan, ${ }^{4}$ Mamas A Mamas. ${ }^{1}$ Farr Institute; ${ }^{2}$ Queen Elizabeth Hospital; ${ }^{3}$ James Cook University Hospital; ${ }^{4}$ Keele Cardiovascular Research Group; * Presenting Author

\subsection{6/heartjnl-2016-309890.34}

Background Over the last decade trans-radial access (TRA) has become more common than trans-femoral access (TFA) for Percutaneous Coronary Intervention (PCI) in the UK. Despite studies highlighting the benefits of this transition, there are concerns that the resulting drop in TFA activity has led to operators and centres losing TFA proficiency, compromising the safety and efficacy of procedures where TFA is necessary. Aims To evaluate the impact of each centre's recent experience of the TFA approach on procedural outcomes in TFA-only procedures.

Methods This retrospective cohort study used procedures recorded in the British Cardiovascular Intervention Society (BCIS) PCI audit from 2007 to 2013 in England and Wales. Centres were split into one of three groups depending on the proportion of total procedures undertaken via TFA in 2013, with patient and procedural characteristics for TFA-only procedures observed within these groups over time. By considering each centre's access site choices in the 12 months prior to procedure date, simply-derived measures were used to capture the 'recent TFA experience' of the operating centre for each procedure. The association of these measures on 30-day mortality, after risk-adjustment, were then studied using multiple logistic regression.

Results A total of 235,474 procedures were available for analysis. Unadjusted TFA-only mortality in centres who were early-adopters of TRA increased more rapidly than for centres who maintained high TFA activity, and this was driven by higher baseline risk. After case-mix adjustment, recent TFA experience was found to have no effect on 30-day mortality $(\mathrm{OR}=0.99$ per 0.1 increase in recent TFA proportion; $\mathrm{CI}=0.96$ to $1.01 ; \mathrm{p}=0.220$ ), with similar results when restricting to procedures with low clinical-complexity $(\mathrm{OR}=0.98$ per 0.1 increase in recent femoral proportion; $\mathrm{CI}=0.95$ to $1.01 ; \mathrm{p}=0.245)$.

Conclusions Poorer TFA outcomes for predominantly high TRA centres are driven by the propensity of these centres to utilise TFA in the highest risk patients. Once differences in case mix are adjusted for, TFA outcomes are similar between high and low radial proportion centres, with no evidence to suggest that increasing unfamiliarity with the TFA technique is detrimental. The outcome gains achieved by the national adoption of TRA is not attenuated by a loss of TFA proficiency, and centres should be encouraged to continue to adopt TRA as the default access site for PCI wherever possible.

\section{THROMBUS ASPIRATION DOES NOT REDUCE MORTALITY IN STEMI PATIENTS: A META-ANALYSIS OF 20,192 PATIENTS, WITH IMPLICATIONS FOR FUTURE TRIAL DESIGN}

Yousif Ahmad*, Sayan Sen, Sukhjinder Nijjer, Daniel Keene, Chris Cook, Ricardo Petraco Matthew Shun-Shin, Graham Cole, Rasha Al-Lamee, Iqbal Malik, Christopher Baker, Ghada Mikhail, Rodney Foale, Jamil Mayet, Justin Davies, Darrel Francis. Imperial College London; *Presenting Author

\subsection{6/heartjnl-2016-309890.35}

Background Thrombus aspiration is a mechanistically logical adjunct in primary angioplasty for acute myocardial infarction. Individual randomised controlled trials (RCTs) have not shown a consensus of mortality reduction and there are concerns about stroke. We perform a meta-analysis of all available RCT data on thrombus aspiration, including the large, recently published TOTAL trial.

Method and results A meta-analysis of RCTs of thrombus aspiration, including the recent TOTAL data was performed. At 30 days (11 trials; 20,192 patients) there was a marginally non-significant reduction in all cause-mortality with thrombus aspiration (OR $0.844,95 \%$ CI $0.710-1.003, p=0.05$ ). The longer-term follow-up data (13 trials; 20,142 patients) was similarly non-significant (OR $0.89,95 \%$ CI $0.78-1.01, \mathrm{p}=$ 0.08). At both 30 days and longer-term follow-up, there was a statistically significant increase in stroke with thrombus aspiration $(\mathrm{OR} 1.56,95 \% \mathrm{CI} 1.05$ to $2.32, \mathrm{p}=0.03$, and $\mathrm{OR}$ $1.94,95 \%$ CI 1.24 to $3.04, \mathrm{p}=0.04$ respectively).

Conclusions The point estimates in the meta-analyses suggest that thrombus aspiration may prevent four deaths per thousand at the cost of two strokes per thousand. Although this 